\title{
Density Regimes of Low-Aspect-Ratio RFP Plasmas in RELAX
}

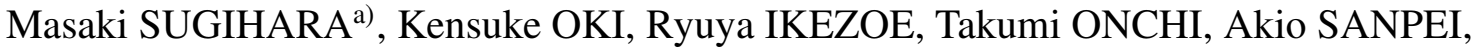 \\ Haruhiko HIMURA, Sadao MASAMUNE ${ }^{\text {b) }}$, Tsuyoshi AKIYAMA ${ }^{1)}$, Akira EJIRI $^{2)}$, \\ Keishi SAKAMOTO ${ }^{3)}$, Kazunobu NAGASAKI ${ }^{4)}$ and Vladimir ZHURAVLEV ${ }^{5)}$ \\ Kyoto Institute of Technology, Matsugaki, Sakyo-ku, Kyoto 606-8585, Japan \\ 1) National Institute for Fusion Science, 322-6 Oroshi-cho, Toki 509-5292, Japan \\ ${ }^{2)}$ The University of Tokyo, 5-1-5 Kashiwanoha, Kashiwa 277-8561, Japan \\ 3) Japan Atomic Energy Agency, 801-1, Mukoyama, Naka 311-0193, Japan \\ ${ }^{4)}$ Kyoto University, Gokasho, Uji 611-0011, Japan \\ ${ }^{5)}$ Kurchatov Institute, Kurchatov-Sq., Moscow 123182, Russia
}

(Received 18 December 2009 / Accepted 19 May 2010)

\begin{abstract}
The line-averaged electron density has been measured over a wide range of discharge conditions in a lowaspect-ratio reversed field pinch (RFP) machine RELAX. A $104 \mathrm{GHz}$ heterodyne interferometer was mainly used throughout the experiments. The measured electron density $n_{e}$ varied from $\sim 0.3 \times 10^{19} \mathrm{~m}^{-3}$ to $\sim 3 \times 10^{19} \mathrm{~m}^{-3}$, depending upon the discharge conditions. A new $60 \mathrm{GHz}$ homodyne interferometer, characterized by simplicity and low cost, has been developed for the density measurement particularly in low density regime. The results from the new homodyne interferometer have shown good agreement with those from the heterodyne system in low-density regime of $n_{\mathrm{e}}$ up to $\sim 10^{19} \mathrm{~m}^{-3}$.
\end{abstract}

(c) 2010 The Japan Society of Plasma Science and Nuclear Fusion Research

Keywords: reversed field pinch, RELAX, millimeter-wave interferometer, electron density measurement

DOI: $10.1585 /$ pfr.5.S2061

\section{Introduction}

The reversed field pinch (RFP) is one of the toroidal confinement systems for high- $\beta$ plasmas. Recent progress has changed a traditional picture of stochastic RFP transport due to multiple tearing modes by establishing two methods of active control of MHD instabilities. One is to suppress the amplitudes of all the modes by inductive edge parallel current drive to realize tearing-stable current density profile. In MST [1] and TPE-RX [2], the pulsed poloidal current drive (PPCD) with ice pellet injection for central fueling has led to improved confinement at total beta value of $\sim 30 \%$. The other is to allow only a single mode to grow, with the resultant closed flux surfaces in a large magnetic island in otherwise stochastic region [3].

The low-aspect-ratio (low- $A$ ) RFP configuration has the following attractive features with a possibility to lead to a new RFP fusion reactor concept [4]. The safety factor $q$ profile shows that the $\mathrm{m}$ (poloidal mode number) $=1$ mode resonant surfaces are less densely spaced in the core region so that a single mode could grow to a large amplitude without interacting the neighboring modes. It might help to attain a single helicity state where we can expect healing of magnetic chaos. In addition, the low-A RFP configuration may realize larger fraction of neoclassical pressure-driven (bootstrap) current than in conventional RFP mainly due to the effect of neoclassical electron viscosity [4].

The RELAX machine [5] was constructed to explore

author'se-mail: ${ }^{\text {a) }}$ sugiha07@nuclear.es.kit.ac.jp, ${ }^{\text {b) }}$ masamune@kit.ac.jp the low-aspect-ratio regime in the RFP configuration. The major research subjects in the initial phase include MHD characteristics, and we have found the possible attainment of helical RFP configuration which may lead to confinement improvement. The electron density is one of the most important parameters characterizing the plasma performance, and we have carried out density measurements with a $104 \mathrm{GHz}$ heterodyne interferometer over a wide range of discharge conditions in RELAX. In what follows, we will discuss the density behavior in RELAX. In addition, a new homodyne interferometer has been developed, and initial results from this interferometer will also be discussed.

\section{Experimental Apparatus}

Figure 1 shows a top view of the RELAX machine [6] with diagnostics arrangement related to the present density studies. The vacuum vessel is made of $4 \mathrm{~mm}$ thick stainless steel (SS) with major radius $R$ of $0.51 \mathrm{~m}$ and minor radius $a$ of $0.25 \mathrm{~m}$. The aspect ratio $A(=R / a)$ is 2.0 , worlds lowest value ever in RFP machines. No conducting shell is attached on the outer surface of the vessel, so, the vessel acts as a resistive boundary. The vessel has two poloidal gaps, one of which is insulated with a dielectric flange, and the other electrically connected with a SS flange. We have attached a pair of feedback-controlled saddle coils at the insulated gap to compensate for the field errors originating from saddle current at the gap. The interferometer was set 


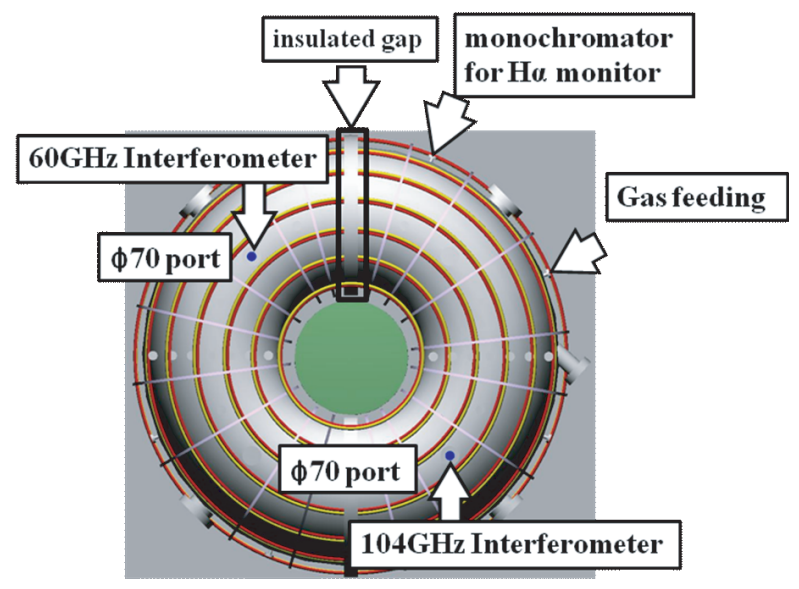

Fig. 1 Top view of RELAX with diagnostics arrangement.

at the location $3 \pi / 4$ away toroidally from the insulated gap. The emission of $\mathrm{H}_{\alpha}$ line was monitored at $\pi / 8$ away from the gap as an indication of localized plasma-wall interaction or recycling of neutral particles.

In the present study, we have used two kinds of interferometers. One is a $104 \mathrm{GHz}$ heterodyne system on loan from the TST group at the University of Tokyo, and the other is a $60 \mathrm{GHz}$ homodyne one developed at Kyoto Institute of Technology. The detailed density measurement has been performed using the heterodyne system consisting of two $(104.00 \mathrm{GHz}$ and $104.88 \mathrm{GHz})$ gun oscillators. The frequency of both the transmitted and reference waves are converted to the intermediate frequency, IF $(880 \mathrm{MHz})$ with mixers. The IF signals are then amplified and are mixed with an IQ detector whose outputs are cosine and sine components of the phase shift caused by the plasma. We should note that the cutoff electron density for $104 \mathrm{GHz}$ is $1.34 \times 10^{20} \mathrm{~m}^{-3}$.

\section{Electron Density Behavior in RELAX}

Electron density measurements have been performed over the following range of discharge parameters where macroscopically stable low- $A$ RFP plasmas have been obtained within the present capability of our power supply system: the plasma current $I_{\mathrm{p}}$ from 40 to $80 \mathrm{kA}$, the filling pressure of hydrogen $p_{\mathrm{f}}$ from 0.1 to $2.0 \mathrm{mTorr}$, and the pinch parameter $\Theta$ (= (edge poloidal field $) /($ average toroidal field $\left.\left\langle B_{\mathrm{t}}\right\rangle\right)$ ) from $\sim 2.0$ to 3.5 , the field reversal ratio $F$ ( = (edge toroidal field) $\left./\left\langle B_{\mathrm{t}}\right\rangle\right)$ from slightly positive $(\sim 0.1)$ to deep reversal of $\sim-1.0$.

Figure 2 shows the time evolutions of $I_{\mathrm{p}}, \mathrm{H}_{\alpha}$ line intensity $I_{\mathrm{H}_{\alpha}}$, and line averaged electron density $n_{\mathrm{e}}$ measured using the $104 \mathrm{GHz}$ interferometer in typical RELAX plasmas. The four different current waveforms correspond to three different values of the filling pressure of $01,0.2$, and 0.4 mTorr, respectively. Two of them are for the same filling pressure of $0.4 \mathrm{~m}$ Torr with different density behaviors. The density evolves almost similarly in the current rising

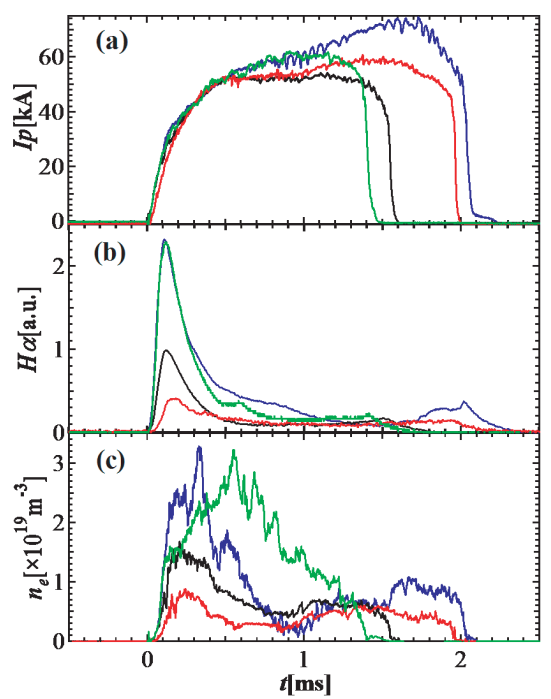

Fig. 2 Typical waveforms of plasma current (a), $H_{\alpha}$ line intensity(b), and line-averaged electron density (d). The colors of the lines represent different filling pressure: red (0.1 mTorr), black ( 0.2 mTorr) , blue (0.4 mTorr), and green $(0.4 \mathrm{mTorr})$, respectively.

phase. Maximum densities at the initial phase are proportional to $p_{\mathrm{f}}$. After that, the density decays to a steady state value in the current flat-top phase. The rapid decay of the density is called density pump out, which results from imbalance of sufficient ionization and insufficient confinement caused by insufficient formation of the RFP configuration. It is typical density behavior in RFPs, and we have observed the similar density behavior in RELAX. Figure 2 also shows that the density evolution depends on the waveform of $I_{\mathrm{p}}$ in that when the current continues rising towards the end of discharge, the density is sustained at higher value. The higher density appears to be caused by enhanced recycling which is indicated by slightly increasing $I_{\mathrm{H}_{\alpha}}$. It should be noted that the higher density in the shot shown by the green line arises from higher $\Theta(\sim 3.2)$ and deeper reversal $(F \sim-1.3)$ discharge regime than in other cases.

The $p_{\mathrm{f}}$ dependencies of the maximum density at the initial phase is shown in Fig. 3(a). It is clear that the maximum density at the initial phase is proportional to $p_{\mathrm{f}}$, which indicates that the initial maximum value is determined not by recycling but by ionization of the filling gas. The following rapid decrease in density appears to be caused by imbalance of sufficient ionization and insufficient confinement caused by insufficient formation of RFP configuration for confinement. In contrast, as shown in Fig. 3 (b), the density in the current flat-top phase scatters even for the same value of $p_{\mathrm{f}}$. It is because of the fact that the density in this phase is determined by recycling neutrals, and the recycling depends upon the current waveform after the initial current rise. As the current grows more, the density gets higher with slightly increasing $I_{\mathrm{H}_{\alpha}}$, an indication of enhanced recycling. The scattering may also be 
attributable at least partly to the present equilibrium control system in RELAX. In the present system, the vertical coil current is pre-programmed so that the waveform similar to that of the plasma current can be produced. Thus the actual controllability depends on the real plasma current waveform. This simplified system restricts the shotto-shot reproducibility even for the fixed discharge conditions. New feedback control systems are under construction for both the equilibrium and magnetic boundary conditions using toroidal and poloidal arrays of saddle coils. It has been confirmed that in RELAX we could attain a wide range of electron density in the current flat-top phase from $1 \times 10^{18} \mathrm{~m}^{-3}$ to $\sim 1.5 \times 10^{19} \mathrm{~m}^{-3}$ by adequately choosing the discharge conditions.

The density regime in the RFP is sometimes discussed in terms of the value $I / N$, where $N$ is the line density (density per unit length in toroidal direction). Figure 4 shows the line density vs. plasma current attained in RELAX, and the reference lines correspond to the values of $I / N$ from $3.1 \times 10^{-14} \mathrm{~A} \cdot \mathrm{m}$ to $20 \times 10^{-14} \mathrm{~A} \cdot \mathrm{m}$. The $I / N$ values in RELAX should be compared with those in other RFP machines; $I / N=2-6 \times 10^{-14} \mathrm{~A} \cdot \mathrm{m}$ in RFX, 4-
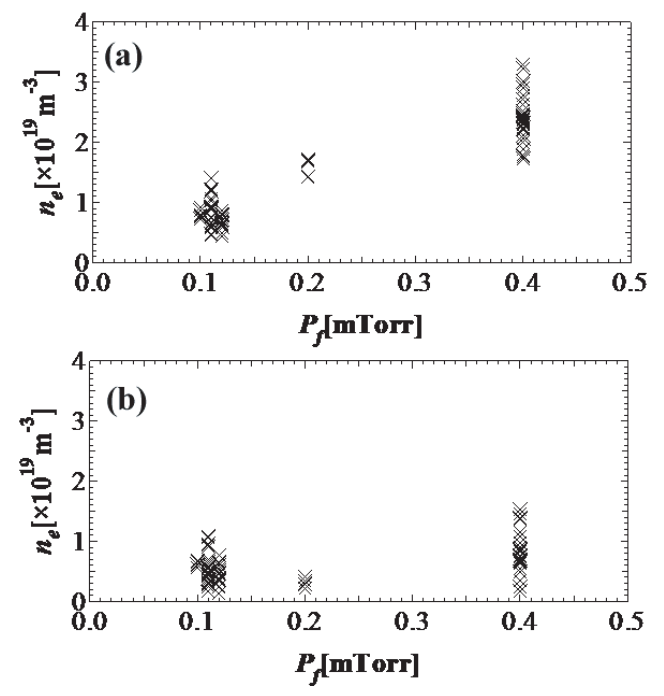

Fig. 3 Pressure dependence of the initial peak density (a) and density at current flat-topped phase (b).

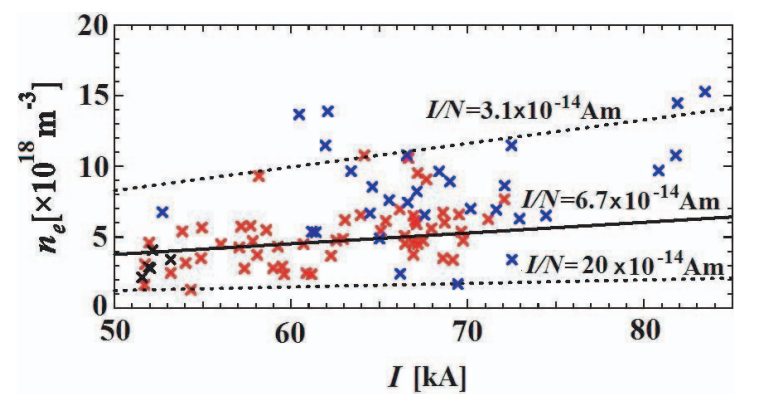

Fig. 4 Attained $I / N$ regime in RELAX under various discharge conditions. The colors represent the filling pressures as in Fig. 2.
$12 \times 10^{-14} \mathrm{~A} \cdot \mathrm{m}$ in MST, $8-20 \times 10^{-14} \mathrm{~A} \cdot \mathrm{m}$ in TPE-RX and $2-15 \times 10^{-14} \mathrm{~A} \cdot \mathrm{m}$ in Extrap-T2 [7]. The higher values of $I / N$ tend to be achievable in meta-wall machines with low recycling, while in FRX, in which graphite tiles covered the whole inner surface, it was difficult to operate at low density. The lower bound of $I / N$ is determined by the achievable highest density. We should note that the comparison of $I / N$ in Fig. 4 is based on standard operation without additional fueling. Recent progress in fueling method such as ice pellet injection may alter the lowest $I / N$. The higher bound of $I / N$ in RELAX may be attributable to the SS wall boundary, while the lower bound, i.e., achievable highest density, has not been made clear yet. The observed wide range of $I / N$ values attained in RELAX may be related on a wide range of operational regions in $F-\Theta$ space [8]. Since magnetic field line stochasticity near the edge depends on the radial location of the field reversal surface, a wide range of operational regions indicates a variety of degrees of recycling even at the same plasma current or filling pressure.

\section{Initial Results Using $60 \mathrm{GHz}$ Homodyne System}

A new $60 \mathrm{GHz}$ homodyne system has been developed for the measurement particularly in low-density regime around $10^{19} \mathrm{~m}^{-3}$. The system is characterized by relatively low cost; it may be applicable to multi-chord density measurements. As shown in Fig. 5, the system consists of a $60 \mathrm{GHz}$ IMPATT oscillator (HUGES, 47174H-1280) whose maximum power is $25.4 \mathrm{dBm}$, and a cross detector which simultaneously detects reference and transmitted waves from two ports. In order to protect the oscillator from reflected wave, an isolator is inserted in the circuit. A directional coupler (HUGES, 45324H-1210) splits the microwave into probe and reference waves. This system is simple and the use of cross detector has lead to improved accuracy in the phase shift measurement.

In Fig. 6, we have compared the Lissajous figure from the $104 \mathrm{GHz}$ heterodyne with that from the $60 \mathrm{GHz}$ homodyne systems. It is clear that the trace is circular for the heterodyne system, while it is deformed elliptically with a shift of its center for the homodyne system. The ellip-

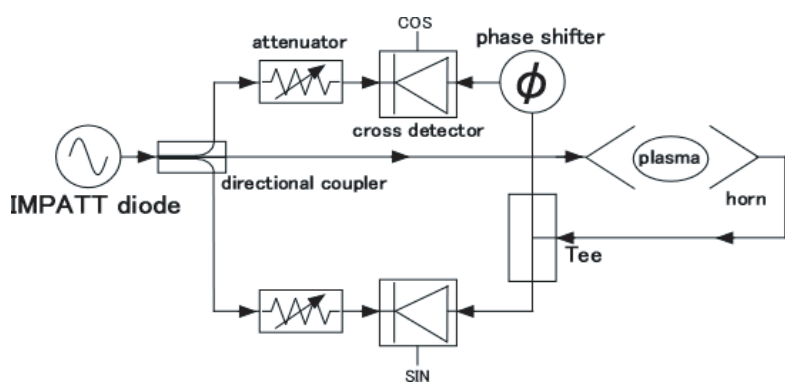

Fig. 5 Block diagram of the homodyne interferometer. 

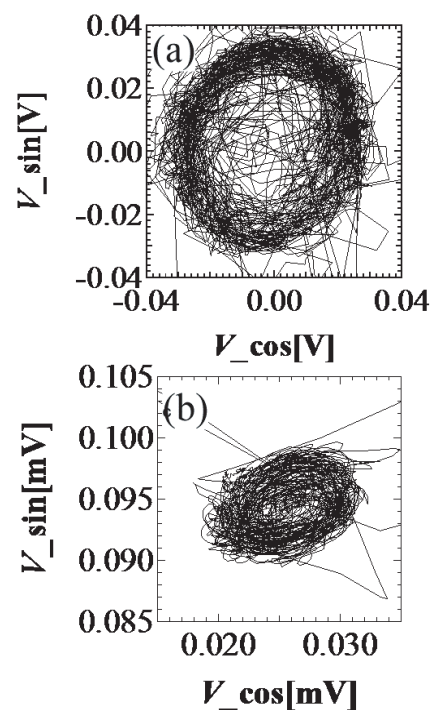

Fig. 6 Lissajous figure from the heterodyne interferometer (a) and the homodyne system (b).

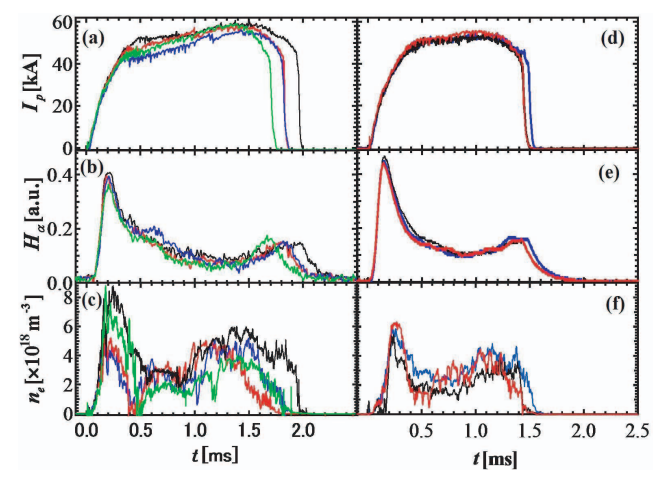

Fig. 7 Comparison of the density evolution measured using the heterodyne ((a), (b), (c)) and homodyne ((d), (e), (f)) interferometers under the same discharge conditions. Note that they are not the simultaneous measurements.

tic deformation is caused by the difference in sensitivity of the detectors, while the shift is due to inevitable DC components in the homodyne system.

The accuracy of the homodyne system has been estimated by comparing the density measurements from two systems, although the results are not from simultaneous measurements. In Fig. 7, we have compared the density evolution from the two interferometers in plasmas with the same discharge conditions ( $p_{\mathrm{f}}=0.1 \mathrm{mTorr}, \Theta \sim 2.8$ and $F \sim-0.8$ ). The time evolution of the density shows the same tendency throughout the discharges, although there are slight differences. Maximum densities at the initial phase are almost the same. There appears to be slight systematic difference of $n_{\mathrm{e}}$, which may be attributable to the difference in the wall conditions, at the current flat-top phase; the total number of discharges from the latest release of vacuum to the density measurement was smaller for the heterodyne interferometer than the case with the homodyne system.

One may recognize the fluctuating components in the density traces. We have carried out statistical analyses of the frequency spectra of the density signals. The fluctuating components in the low frequency region $(f<50 \mathrm{kHz})$ have no significant difference between the two interferometers. It suggests that the low-frequency components are probably related with MHD phenomena in the plasma. The high frequency components $(f>100 \mathrm{kHz})$ are 2-3 times higher for the homodyne system, suggesting that the highfrequency components include noise signals. It has been demonstrated that the newly developed homodyne interferometer can be applied to the density measurement in the range up to $\sim 10^{19} \mathrm{~m}^{-3}$.

\section{Conclusion}

The electron density measurement has been carried out in a low-A RFP machine RELAX over a wide range of discharge conditions using the $104 \mathrm{GHz}$ heterodyne and $60 \mathrm{GHz}$ homodyne interferometers. It has been found that the electron density is in the range from $0.3 \times 10^{19} \mathrm{~m}^{-3}$ to $\sim 3 \times 10^{19} \mathrm{~m}^{-3}$ depending on the discharge conditions. From the measurement of line-averaged electron density, it is found that the $I / N$ value of the normal RFP discharges in RELAX is about $3-20 \times 10^{-14} \mathrm{~A} \cdot \mathrm{m}$, which is wider in range than in other RFP plasmas. It may be related to the wide range of operational regime of RELAX plasmas in $F-\Theta$ space. The newly developed $60 \mathrm{GHz}$ homodyne interferometer, characterized by simplicity and low cost, has been shown to be applicable to RELAX particularly in the low density regime.

\section{Acknowledgment}

This work was supported by the NIFS collaboration program (NIFS09KCHH028).

[1] M. D. Wyman et al., Phys. Plasmas 15, 010701 (2008).

[2] H. Koguchi et al., Plasma Fusion Res. 4, 022 (2009).

[3] R. Lorenzini et al., Nature Physics 5, 570 (2009).

[4] S. Shiina et al., Phys. Plasmas 12, 080702 (2005).

[5] S. Masamune et al., J. Phys. Soc. Jpn. 76, 123501 (2007).

[6] S. Masamune et al., J. Fusion Energy 28, 187 (2009).

[7] Y. Yagi et al., Fusion Energy 2000 (Proc. 18th IAEA Fusion Energy Conference), IAEA Vienna, EX4/6, 2000.

[8] R. Ikezoe et al., Plasma Fusion Res. SERIES 8, 1031 (2009). 